\title{
ON THE ABSOLUTE VALUE OF THE $S O(3)$-INVARIANT AND OTHER SUMMANDS OF THE TURAEV-VIRO INVARIANT
}

\author{
MAXIM SOKOLOV \\ George Washington University \\ Funger 428A \\ $2201 \mathrm{G}$ St., $N W$ \\ Washington, D.C., 20052, U.S.A. \\ E-mail:sokolov@math.gwu.edu
}

1. Introduction. It was proved in [S1] and [S2] that each Turaev-Viro invariant $T V(M)_{q}$ for a 3 -manifold $M$ is a sum of three invariants $T V_{0}(M)_{q}, T V_{1}(M)_{q}$, and $T V_{2}(M)_{q}$ (for definition of the Turaev-Viro invariants, see [TV]). It follows from the Turaev-Walker theorem (see [T1], [W]) that if $q^{2}$ is a primitive root of unity of an odd degree then, up to normalization, $T V_{0}(M)_{q}$ coincides with the square of the modulus of the so-called $S O(3)$-invariant $\tau_{e}(M)$ defined in [T2]. For a connection between $S O$ (3)-invariants and the Reshetikhin-Turaev invariants, see [KM] and [BHMV].

With a help of suitable normalizations we make the numbers $\left(T V_{0}(M)_{q}+T V_{2}(M)_{q}\right)$, $T V_{0}(M)_{q}$, and $T V_{1}(M)_{q}$ to be invariant under removing of 3-balls. That allows us to define these three invariants on a triangulation of a closed 3-manifold $M$.

It is natural to relate the invariants $T V_{N}(M), N=0,1,2$, to the Turaev-Viro invariants. Here we show that for every 3-manifold $M$ the following holds:

$$
\begin{gathered}
T V_{0}(M)_{q}+T V_{2}(M)_{q}=\frac{1}{2}\left(T V(M)_{q}+T V(M)_{-q}\right), \\
T V_{1}(M)_{q}=\frac{1}{2}\left(T V(M)_{q}-T V(M)_{-q}\right) .
\end{gathered}
$$

At the end of the paper we present a few tables. There are a lot of numerical tables of the Turaev-Viro and Reshetikhin-Turaev invariants (see, for instance, [KL1], [KL2], $[\mathrm{N}],[\mathrm{S} 2])$. An advantage of our tables is that the values of the invariants are presented as polynomials in $q$ with integer coefficients.

1991 Mathematics Subject Classification: Primary 57N10; Secondary 57M20.

Research is partially supported by INTAS, grant 94201.

The paper is in final form and no version of it will be published elsewhere. 
The paper was written when I was a student of S. V. Matveev at the Cheljabinsk State University, Russia. I am very grateful also to R. Zh. Aleev and C. Blanchet for many useful conversations. An interesting question of D. Yetter was a reason for appearing of section 5. A. Macovetsky helped me to prove lemma 3 in section 6. I am greatly indebted to G. Masbaum who helped to find some deficiencies in the first version of the paper. My special thanks to the Organizing committee of the mini-semester on Knot theory in Warsaw, July-August 1995.

2. Simple polyhedra and their local moves. A 2-dimensional polyhedron $X$ is called simple if the link of any point of $X$ is homeomorphic to one of the following polyhedra: (1) a circle, (2) a circle with two radii, (3) a circle with three radii, (4) the segment $[0,1],(5)$ a wedge of three segments with a common endpoint.

The set of points of a simple polyhedron $X$ having links of types (4) or (5) is called a boundary of $X$ and denoted by $\partial X$. The points with links of type (3) are called vertices of $X$. By an edge of $X$ we mean a connected component of the set of points having the links of type (2).

Simple polyhedra are also called fake surfaces. This class of polyhedra generates the class of special polyhedra. Recall that a simple polyhedron $X$ is called special if $\partial X=\emptyset$ and each 2-component of $X$ is a 2-cell.

A simple polyhedron $X$ with $\partial X=\emptyset$ is called a simple spine of a compact 3-manifold $M$ with $\partial M \neq \emptyset$ if there exists an embedding $i: X \rightarrow M$ such that $M \searrow i(X)$, i.e. $M$ collapses onto $i(X)$. In the case of a closed $M$, a polyhedron $X$ is called a simple spine of $M$ if it is a simple spine of $M$ with an open 3-ball removed. A simple spine is called special if it is a special polyhedron. It is known that every compact connected 3 -manifold has a special spine (see $[\mathrm{Ca}],[\mathrm{M}])$.

Let us describe now special polyhedra-with-boundary $P_{1}, \ldots, P_{4}$. Let $P_{1}$ be the polyhedron obtained from a disk $D^{2}$ by attaching two semidisks along two parallel chords, $h_{1}$ and $h_{2}$ of $D^{2}$. The polyhedron $P_{2}$ is obtained from $D^{2}$ by attaching a semidisk along $h_{2}$ and the second one along a simple curve $l$ in $D^{2}$ that has the same endpoints as $h_{1}$ and intersects $h_{2}$ transversally in exactly two points. Let $R=R_{1} \cup R_{2} \cup R_{3}$ be a triod consisting of three radii of the disk $D^{2}$. The polyhedron $P_{3}$ is obtained from the polyhedron $\left(D^{2} \times\{0\}\right) \cup(R \times I)$ by attaching a semidisk along a chord $h_{1} \subset D^{2}$ that intersects the radius $R_{1}$ in just one interior point. The polyhedron $P_{4}$ is obtained from $\left(D^{2} \times\{0\}\right) \cup(R \times I)$ by attaching a semidisk along a simple curve that has the same endpoints as $h_{1}$ and intersects the triod $R$ in exactly two points, on $R_{2}$ and $R_{3}$.

By $\mathcal{L}$-move on simple polyhedra we mean a replacement of a fragment homeomorphic to $P_{1}$ by $P_{2}$. By $\mathcal{M}$-move on simple polyhedra we mean a replacement of a fragment homeomorphic to $P_{3}$ by $P_{4}$ (for details, see $[\mathrm{M}],[\mathrm{P}]$ ).

Let a circle $c$ bound a 2-disk in a 2-component of a special polyhedron $X$. By $\mathcal{B}$-move we mean an attaching of additional 2-disk to $X$ along $c$ (for details, see [TV]).

It is proved in $[\mathrm{M}]$ that any two special spines of a 3-manifold can be transformed one to another by a sequence of the moves $\mathcal{M}^{ \pm 1}$ and $\mathcal{L}^{ \pm 1}$. Note, that applying $\mathcal{L}$ several times, 
one can transform any simple spine into a special one. So the theorem of S. V. Matveev is true for simple spines too.

The $\mathcal{B}$-move on a simple spine of a 3 -manifold $M$ corresponds to removing of one 3-ball from $M$.

3. The Turaev-Viro invariants. Throughout the paper, let us fix $r \geq 3$ and a root of unity $q$ of degree $2 r$ such that $q^{2}$ is a primitive root of degree $r$.

In this section we recall how V. G. Turaev and O. Y. Viro define their invariants on a simple polyhedron $X$ (cf. [TV]). Let $v_{1}, \ldots, v_{d}$ be the vertices of $X$, let $e_{1}, \ldots, e_{f}$ be the edges of $\partial X$ and let $\Gamma_{1}, \ldots, \Gamma_{b}$ be the 2-components of $X$.

By a coloring of $X$ we mean an arbitrary mapping

$$
\varphi:\left\{\Gamma_{1}, \ldots, \Gamma_{b}\right\} \rightarrow \mathbf{Z}_{r-1}=\{0,1, \ldots, r-2\} .
$$

A triple $(i, j, k) \in \mathbf{Z}_{r-1}^{3}$ will be called admissible if

$$
\begin{gathered}
2 r-4 \geq i+j+k \equiv 0 \quad(\bmod 2), \\
|i-j| \leq k \leq i+j .
\end{gathered}
$$

A coloring $\varphi$ is called admissible if for any edge $E$ of $X-\partial X$ the colors of the 2-components incident to $E$ form an admissible triple. Let us denote the set of admissible triples by $a d m$ and the set of admissible colorings of $X$ by $\operatorname{Adm}(X)$.

By a coloring of a regular graph $G$ we shall mean any mapping of the set of its edges to $\mathbf{Z}_{r-1}$. Let us denote the set of colorings of $X$ by $\operatorname{Col}(X)$. Any coloring $\varphi$ of a simple polyhedron $X$ induces in a natural way a coloring $\partial \varphi$ of its boundary $\partial X$ : an edge of $\partial X$ takes the color of the 2-component of $X$ in whose boundary this edge is contained.

Let $\Gamma_{i}, \Gamma_{j}, \Gamma_{k}$ be 2-components incident to an edge $E$ of $X$ and let $\varphi \in \operatorname{Adm}(X)$. We shall say that an unordered triple $\left\{\varphi\left(\Gamma_{i}\right), \varphi\left(\Gamma_{j}\right), \varphi\left(\Gamma_{k}\right)\right\}$ is a color of the edge $E$. There are six wings incident to any vertex $v$ of a simple polyhedron. Suppose they receive under $\varphi$ the values $i, j, k, l, m, n \in \mathbf{Z}_{r-1}$. A 6 -tuple $\left(\begin{array}{ccc}i & j & k \\ l & m & n\end{array}\right)$ is called a color of the vertex $v$ if $\{i, j, k\}$ is a color of some edge incident to $v$ and $(i, l),(j, m),(k, n)$ are the colors of opposite 2-components incident to $v$.

For an integer $n>0$ set

$$
\begin{gathered}
{[n]_{q}=\frac{q^{n}-q^{-n}}{q-q^{-1}},} \\
{[n]_{q} !=[n]_{q}[n-1]_{q} \ldots[2]_{q}[1]_{q} .}
\end{gathered}
$$

Set also $[0]_{q}=[0]_{q} !=1$. For a color $\{i, j, k\}$ of an edge set

$$
\Delta_{q}(i, j, k)=\left(\frac{[\underline{i}+\underline{j}-\underline{k}]_{q} ![\underline{i}+\underline{k}-\underline{j}]_{q} ![\underline{j}+\underline{k}-\underline{i}]_{q} !}{[\underline{i}+\underline{j}+\underline{k}+1]_{q} !}\right)^{1 / 2}
$$

where $\underline{i}=i / 2$. Note that the expression in the round brackets presents a real number. By the square root $x^{1 / 2}$ of a real number $x$ we mean the positive root of $|x|$ multiplied by $\sqrt{-1}$ if $x<0$. 

formula

Let $\left(\begin{array}{ccc}i & j & k \\ l & m & n\end{array}\right)$ be a color of some vertex $v$. A symbol of $v$ is defined by the following

$$
\begin{aligned}
\left|T_{v}^{\varphi}\right|_{q}=\left|\begin{array}{ccc}
i & j & k \\
l & m & n
\end{array}\right|_{q}=(\sqrt{-1})^{-(i+j+k+l+m+n)} \Delta_{q}(i, j, k) \Delta_{q}(i, m, n) \times \\
\quad \times \Delta_{q}(j, l, n) \Delta_{q}(k, l, m)\left[\begin{array}{ccc}
i & j & k \\
l & m & n
\end{array}\right]_{q}
\end{aligned}
$$

where

$$
\begin{aligned}
{\left[\begin{array}{ccc}
i & j & k \\
l & m & n
\end{array}\right]_{q}=\sum_{z}(-1)^{z}[z+1]_{q} !\left\{[z-\underline{i}-\underline{j}-\underline{k}]_{q} ![z-\underline{i}-\underline{m}-\underline{n}]_{q} ![z-\underline{j}-\underline{l}-\underline{n}]_{q} ![z-\underline{k}-\underline{l}-\underline{m}]_{q} ! \times\right.} \\
\left.\quad \times[\underline{i}+\underline{j}+\underline{l}+\underline{m}-z]_{q} ![\underline{i}+\underline{k}+\underline{l}+\underline{n}-z]_{q} ![\underline{j}+\underline{k}+\underline{m}+\underline{n}-z]_{q} !\right\}^{-1} .
\end{aligned}
$$

Here $z$ runs through the non-negative integers such that all expressions in the square brackets are non-negative. For $i \in \mathbf{Z}_{r-1}$ put

$$
w_{i, q}=(\sqrt{-1})^{i}[i+1]_{q}^{1 / 2} .
$$

For $\varphi \in \operatorname{Adm}(X)$ put

$$
|X, \varphi|_{q}=\prod_{i=1}^{b} w_{\varphi\left(\Gamma_{i}\right), q}^{2 \chi\left(\Gamma_{i}\right)} \prod_{s=1}^{f} w_{\partial \varphi\left(e_{s}\right), q}^{\chi\left(e_{s}\right)} \prod_{j=1}^{d}\left|T_{v_{j}}^{\varphi}\right|_{q}
$$

where $\chi$ is the Euler characteristic (the 2-components of $X$ and the edges of $\partial X$ are thought to be open, so if $e_{s}$ is homeomorphic to $\mathbf{R}$ then $\chi\left(e_{s}\right)=-1$ and if $e_{s}$ is homeomorphic to $S^{1}$ then $\left.\chi\left(e_{s}\right)=0\right)$.

The Turaev-Viro invariant for the simple polyhedron $X$ is given by

$$
T V(X)_{q}=\sum_{\varphi \in \operatorname{Adm}(X)}|X, \varphi|_{q}
$$

It is proved in [TV] that $T V(X)_{q}$ is invariant under moves $\mathcal{L}^{ \pm 1}$ and $\mathcal{M}^{ \pm 1}$. It follows from Matveev's theorem that if $X$ is a simple spine of a 3-manifold $M$ then $T V(M)_{q}=T V(X)_{q}$ is a topological invariant of $M$.

Note that in [TV] a different normalization is used. The original Turaev-Viro invariant is given by the formula

$$
T V^{*}(X)_{q}=\omega^{-2 \chi(X)+\chi(\partial X)} T V(X)_{q},
$$

where $\omega=\sqrt{2 r} /\left|q-q^{-1}\right|$. It is proved in [TV] that $T V^{*}(X)_{q}$ is invariant under $\mathcal{B}^{ \pm 1}$ also.

Remark 1. It is easily seen that if $q$ is a primitive root of unity of degree $2 r$ and $\partial X=\emptyset$ then the numbers $|X, \varphi|_{q}$, and therefore the numbers $T V(X)_{q}$ and $T V^{*}(X)_{q}$, lie in $\mathbf{Q}(q)$.

4. The summand-invariants. The set of 2-components of $X$ that receive odd colors under a coloring $\varphi \in A d m(X)$ forms a surface embedded in $X$. We denote this surface by $S(\varphi)$. Note that $\partial S(\varphi) \subseteq \partial X$.

Present the set $\operatorname{Adm}(X)$ as a disjoint union of subsets $\operatorname{Adm}_{0}(X), \operatorname{Adm}_{1}(X)$ and $A d m_{2}(X)$, where 
0) $\varphi \in A d m_{0}(X) \Leftrightarrow(\varphi \in \operatorname{Adm}(X)) \&(S(\varphi)=\emptyset)$;

1) $\varphi \in A d m_{1}(X) \Leftrightarrow(\varphi \in \operatorname{Adm}(X)) \&(\chi(S(\varphi)) \equiv 1 \quad(\bmod 2))$;

2) $\varphi \in A d m_{2}(X) \Leftrightarrow(\varphi \in A d m(X)) \&(S(\varphi) \neq \emptyset) \&(\chi(S(\varphi)) \equiv 0 \quad(\bmod 2))$.

For any coloring $\alpha$ of $\partial X$ and $N \in\{0,1,2\}$ put

$$
\Omega_{N}(X, \alpha)_{q}=\sum_{\substack{\varphi \in \operatorname{Adm}_{N}(X) \\ \partial \varphi=\alpha}}|X, \varphi|_{q} .
$$

If $\left\{\varphi \in A d m_{N}(X): \partial \varphi=\alpha\right\}=\emptyset$, then $\Omega_{N}(X, \alpha)_{q}=0$. Put also

$$
T V_{N}(X)_{q}=\sum_{\alpha \in \operatorname{Col}(\partial X)} \Omega_{N}(X, \alpha)_{q}
$$

where sum is taken over all colorings of $\partial X$.

Remark 2. $T V(X)_{q}=T V_{0}(X)_{q}+T V_{1}(X)_{q}+T V_{2}(X)_{q}$.

Remark 3. If $q$ is a primitive root of unity of degree $2 r$ then for a simple polyhedron $X$ with $\partial X=\emptyset$ we have $T V_{N}(X)_{q} \in \mathbf{Q}(q)$, for any $N \in\{0,1,2\}$ (see remark 1).

Lemma 1. Let a simple polyhedron $X$ be the union of simple polyhedra $Y$ and $Z$ and let each connected component of $T=Y \cap Z$ be a connected component of both $\partial Y$ and $\partial Z$. Then for any coloring $\beta$ of $\partial X$ we have

$$
\begin{aligned}
& \Omega_{0}(X, \beta)_{q}=\sum_{\alpha \in \operatorname{Col}(T)} \Omega_{0}\left(Y, \alpha \cup\left(\left.\beta\right|_{Y \cap \partial X}\right)\right)_{q} \Omega_{0}\left(Z, \alpha \cup\left(\left.\beta\right|_{Z \cap \partial X}\right)\right)_{q}, \\
& \Omega_{1}(X, \beta)_{q}=\sum_{\substack{\alpha \in \operatorname{Col}(T) \\
K+L \equiv 1(2)}} \Omega_{K}\left(Y, \alpha \cup\left(\left.\beta\right|_{Y \cap \partial X}\right)\right)_{q} \Omega_{L}\left(Z, \alpha \cup\left(\left.\beta\right|_{Z \cap \partial X}\right)\right)_{q}, \\
& \Omega_{2}(X, \beta)_{q}=\sum_{\substack{\alpha \in \operatorname{Col}(T) \\
K+L=2 \text { or } 4}} \Omega_{K}\left(Y, \alpha \cup\left(\left.\beta\right|_{Y \cap \partial X}\right)\right)_{q} \Omega_{L}\left(Z, \alpha \cup\left(\left.\beta\right|_{Z \cap \partial X}\right)\right)_{q} .
\end{aligned}
$$

Proof. This follows from the equalities

$$
|X, \varphi|_{q}=\left|Y,\left(\left.\varphi\right|_{Y}\right)\right|_{q} \cdot\left|Z,\left(\left.\varphi\right|_{Z}\right)\right|_{q},
$$

where $\varphi \in \operatorname{Adm}(X)$ (see Lemma 4.2.A in [TV]), and $\chi(X)=\chi(Y)+\chi(Z)$.

ThEOREM 1. Let $X$ be a simple 2-polyhedron and $\alpha$ be a coloring of $\partial X$. Then for any $N \in\{0,1,2\}$ the number $\Omega_{N}(X, \alpha)_{q}$ is invariant under $\mathcal{L}^{ \pm 1}$ and $\mathcal{M}^{ \pm 1}$.

Pr o of. Let us show that the number $\Omega_{N}(X, \alpha)_{q}$ is invariant under $\mathcal{L}$. The case of $\mathcal{M}$ move is similar. By lemma 1 it is sufficient to prove that $\Omega_{N}\left(P_{1}, \gamma\right)_{q}=\Omega_{N}\left(P_{2}, \gamma\right)_{q}$ for any $N \in\{0,1,2\}$, where $P_{1}$ and $P_{2}$ are the polyhedra from the definition of the $\mathcal{L}$-move and $\gamma$ is a coloring of the graph $\partial P_{1}=\partial P_{2}$. It is easy to check that for any $\gamma$ there is a unique $K \in\{0,1,2\}$ such that $\left\{\varphi \in \operatorname{Adm}\left(P_{i}\right): \partial \varphi=\gamma\right\} \subset A d m_{K}\left(P_{i}\right)$, for $i=1,2$. Therefore

$$
\Omega_{N}\left(P_{1}, \gamma\right)_{q}=\sum_{\substack{\varphi \in \operatorname{Adm}\left(P_{1}\right) \\ \partial \varphi=\gamma}}\left|P_{1}, \varphi\right|_{q} \text { and } \Omega_{N}\left(P_{2}, \gamma\right)_{q}=\sum_{\substack{\psi \in \operatorname{Adm}\left(P_{2}\right) \\ \partial \psi=\gamma}}\left|P_{2}, \psi\right|_{q}
$$

if $N=K$, and $\Omega_{N}\left(P_{1}, \gamma\right)_{q}=\Omega_{N}\left(P_{2}, \gamma\right)_{q}=0$ if $N \neq K$. It is proved in Lemma 4.4.A of $[\mathrm{TV}]$ that the sums are equal. 
Corollary 1. Let $X$ be a simple spine of a 3-manifold $M$. Then $T V_{N}(M)_{q}=$ $T V_{N}(X)_{q}$ is an invariant of $M$ for any $N \in\{0,1,2\}$.

5. The summand-invariants and triangulation. The summand invariants are not invariants under $\mathcal{B}$-move. This prevents us from defining these invariants on a triangulation of a 3 -manifold. Here we modify the invariants $T V_{0}, T V_{1}$, and $T V_{0}+T V_{2}$ to make them invariant under removing of 3-balls.

Put $\omega_{0}=\sqrt{r} /\left|q-q^{-1}\right|$ and $\omega=\sqrt{2 r} /\left|q-q^{-1}\right|$. Let $X$ be a simple polyhedron. Put

$$
\begin{aligned}
& \Omega_{0}^{*}(X, \alpha)_{q}=\omega_{0}^{-2 \chi(X)+\chi(\partial X)} \Omega_{0}(X, \alpha)_{q}, \\
& \Omega_{1}^{*}(X, \alpha)_{q}=\omega^{-2 \chi(X)+\chi(\partial X)} \Omega_{1}(X, \alpha)_{q},
\end{aligned}
$$

and

$$
\Omega_{e}^{*}(X, \alpha)_{q}=\omega^{-2 \chi(X)+\chi(\partial X)}\left(\Omega_{0}(X, \alpha)_{q}+\Omega_{2}(X, \alpha)_{q}\right) .
$$

Lemma 2. Let $X$ be a simple 2-polyhedron and $\alpha$ be a coloring of $\partial X$. Then the numbers $\Omega_{0}^{*}(X, \alpha)_{q}, \Omega_{1}^{*}(X, \alpha)_{q}$, and $\Omega_{e}^{*}(X, \alpha)_{q}$ are invariant under $\mathcal{B}$.

Proof. It follows immediately from the definition of the number $|X, \varphi|_{q}$ that the number $\Omega_{0}^{*}(X, \alpha)_{q}$ is invariant under $\mathcal{B}$ if

$$
\omega_{0}^{2}=w_{j}^{-2} \sum_{\substack{k, l \equiv 0(2) \\ k, l:\{j, k, l\} \in a d m}} w_{k}^{2} w_{l}^{2}
$$

for any even $j \in \mathbf{Z}_{r-1}$, and $\Omega_{1}^{*}(X, \alpha)_{q}, \Omega_{e}^{*}(X, \alpha)_{q}$ are invariant under $\mathcal{B}$ if

for any $j \in \mathbf{Z}_{r-1}$.

$$
\omega^{2}=w_{j}^{-2} \sum_{k, l:\{j, k, l\} \in a d m} w_{k}^{2} w_{l}^{2}
$$

The second equality is proved in $[\mathrm{TV}]$. The proof of the first one is similar. First of all, let us check that

$$
w_{j}^{-2} \sum_{\substack{k, l \equiv 0(2) \\ k, l:\{j, k, l\} \in a d m}} w_{k}^{2} w_{l}^{2}=w_{0}^{-2} \sum_{\substack{s \equiv 0(2) \\ 0 \leq s \leq r-2}} w_{s}^{4}
$$

for any even number $j \in \mathbf{Z}_{r-1}$.

Let $T$ be a polyhedron obtained from a disk $D^{2}$ by attaching one semidisk along a diameter of $D^{2}$. The polyhedron $T$ consists of three 2-cells $\Gamma_{1}, \Gamma_{2}, \Gamma_{3}$. Let the polyhedron $T_{i}$ be obtained from $T$ by attaching a 2-disk along a circle that belongs to the 2 -cell $\Gamma_{i}$, where $i=1$ or 2 .

For any $j \in \mathbf{Z}_{r-1}$ we define a coloring $\beta$ of $\partial T_{1}$ and $\partial T_{2}$ as follows: $\beta\left(\Gamma_{1}\right)=\beta\left(\Gamma_{3}\right)=j$, $\beta\left(\Gamma_{2}\right)=0$.

By definition, we have

$$
\begin{aligned}
& \Omega_{0}\left(T_{1}, \beta\right)_{q}=w_{0}^{2} w_{j}^{2} \sum_{\substack{k, l \equiv 0(2) \\
k, l:\{j, k, l\} \in a d m}} w_{k}^{2} w_{l}^{2}, \\
& \Omega_{0}\left(T_{2}, \beta\right)_{q}=w_{j}^{4} \sum_{\substack{s \equiv 0(2) \\
0 \leq s \leq r-2}} w_{s}^{4} .
\end{aligned}
$$


Note that $T_{1}$ and $T_{2}$ are connected by $\mathcal{L}$-move, therefore $\Omega_{0}\left(T_{1}, \alpha\right)_{q}=\Omega_{0}\left(T_{2}, \alpha\right)_{q}$. This gives us the equality $(*)$.

Clearly, $w_{0}=1$. Thus we have to prove that

$$
\sum_{t=0}^{[r / 2]-1} w_{2 t}^{4}=-r /\left(q-q^{-1}\right)^{2} .
$$

The proof of this equality is straightforward.

Corollary 2. Let $X$ be a simple spine of a 3-manifold $M$. Then the numbers $T V_{0}^{*}(M)_{q}=\Omega_{0}^{*}(X)_{q}, T V_{1}^{*}(M)_{q}=\Omega_{1}^{*}(X)_{q}$, and $T V_{e}^{*}(M)=\Omega_{e}^{*}(X)_{q}$ are invariants of $M$ under removing of 3-balls.

We can define the invariants $T V_{0}^{*}, T V_{1}^{*}$, and $T V_{e}^{*}$ on a triangulation of a 3 -manifold $M$ like the Turaev-Viro invariants were defined in [TV]. For simplicity we will restrict ourselves to the case of closed 3-manifolds only.

Let $M$ be a closed triangulated 3-manifold. Let $a$ be the number of vertices of $M$, let $E_{1}, \ldots, E_{b}$ be the edges of $M$, and let $T_{1}, \ldots, T_{d}$ be the 3 -simplexes of $M$. By a coloring of $M$ we mean an arbitrary mapping $\varphi:\left\{E_{1}, \ldots, E_{b}\right\} \rightarrow \mathbf{Z}_{r-1}$. A coloring $\varphi$ of $M$ is called admissible if for any 2-simplex $A$ of $M$ the colors of the three edges of $A$ form an admissible triple. Denote the set of admissible colorings of $M$ by $\operatorname{Adm}(M)$. We will denote by $A d m_{0}(M)$ the set of admissible colorings of $M$ by even numbers, and by $\operatorname{Adm}_{e}(M)$ the set of admissible colorings of $M$ such that

$$
v-t+f \equiv 0 \quad(\bmod 2),
$$

where $v$ is the number of 3 -simplexes containing an edge colored by an odd number, $t$ is the number of 2-simplexes containing an edge colored by an odd number, and $f$ is the number of edges colored by odd numbers. Note that $\operatorname{Adm}_{0}(M) \subset \operatorname{Adm_{e}}(M)$. Set $A d m_{1}(M)=A d m(M)-A d m_{e}(M)$. A 6-tuple $\left(\begin{array}{ccc}i & j & k \\ l & m & n\end{array}\right)$ is called a color of a 3-simplex $T_{s}$ if $i, j, k$ are the colors of edges of some 2 -face of $T_{s}$ and $(i, l),(j, m),(k, n)$ are the pairs of colors of opposite edges of $T_{s}$. Let

$$
\left|T_{s}^{\varphi}\right|_{q}=\left|\begin{array}{ccc}
i & j & k \\
l & m & n
\end{array}\right|_{q} .
$$

For $\varphi \in \operatorname{Adm}(M)$ put

$$
|M, \varphi|_{q}=\prod_{i=1}^{b} w_{\varphi\left(E_{i}\right), q}^{2} \prod_{s=1}^{d}\left|T_{s}^{\varphi}\right|_{q} .
$$

Proposition 1. For any closed triangulated 3-manifold $M$ we have

$$
\begin{aligned}
& T V_{0}^{*}(M)_{q}=\omega_{0}^{-2 a} \sum_{\varphi \in A d m_{0}(M)}|M, \varphi|_{q}, \\
& T V_{1}^{*}(M)_{q}=\omega^{-2 a} \sum_{\varphi \in A d m_{1}(M)}|M, \varphi|_{q}, \\
& T V_{e}^{*}(M)_{q}=\omega^{-2 a} \sum_{\varphi \in A d m_{e}(M)}|M, \varphi|_{q} .
\end{aligned}
$$


Proof. Let $X$ be the union of the closed barycentric stars of the edges of $M$. It is obvious that $X$ is a special polyhedron. By a finite number of $\mathcal{M}^{ \pm 1}, \mathcal{L}^{ \pm 1}$ and $\mathcal{B}^{-1}$ moves on $X$ we get a simple spine of $M$. Each coloring $\varphi$ of $M$ induces a dual coloring $\varphi^{*}$ of $X$, and it is easy to check that $|M, \varphi|_{q}=\left|X, \varphi^{*}\right|_{q}$ and $\chi(X)=a$, which establishes the formulas.

6. The values of the summand-invariants. Here we express the numbers $T V_{0}(M)_{q}+T V_{2}(M)_{q}$ and $T V_{1}(M)_{q}$ via the Turaev-Viro invariants.

Let $X$ be a special polyhedron. Fix a number $r \geq 3$ and a coloring $\varphi \in \operatorname{Adm}(X)$. A vertex $v$ of the colored polyhedron $X$ is called a switch-vertex if the sum of all odd numbers in the color of $v$ is congruent to 2 modulo 4 .

Lemma 3. Let $X$ be a special polyhedron. Then for any $\varphi \in \operatorname{Adm}(X)$ we have

$$
|X, \varphi|_{q}=(-1)^{\chi(S(\varphi))+x}|X, \varphi|_{-q},
$$

where $x$ is the number of the switch-vertices of $X$.

Proof. It is easy to see that

$$
\begin{gathered}
{[n]_{q}=(-1)^{n-1}[n]_{-q},} \\
{[n]_{q} !=(-1)^{n(n-1) / 2}[n]_{-q} !} \\
w_{i, q}^{2}=(-1)^{i} w_{i,-q}^{2}, \\
\Delta_{q}^{2}(i, j, k)= \begin{cases}\Delta_{-q}^{2}(i, j, k), & \text { if } i, j, k \text { are even } \\
-\Delta_{-q}^{2}(i, j, k), & \text { otherwise. }\end{cases}
\end{gathered}
$$

Let $\left(\begin{array}{ccc}i_{1} & i_{2} & i_{3} \\ i_{4} & i_{5} & i_{6}\end{array}\right)$ be a color of a vertex $v$ of $X$ under $\varphi$. Then we have

$$
(* *) \quad\left[\begin{array}{lll}
i_{1} & i_{2} & i_{3} \\
i_{4} & i_{5} & i_{6}
\end{array}\right]_{q}=(-1)^{\frac{1}{2} \sum_{s, t=1}^{6} i_{s} i_{t}}\left[\begin{array}{ccc}
i_{1} & i_{2} & i_{3} \\
i_{4} & i_{5} & i_{6}
\end{array}\right]_{-q} .
$$

There are three possibilities for the color of $v$.

1) Each number in the color of $v$ is even (even vertex). Then the sign in $(* *)$ is plus.

2) There are four odd numbers in the color of $v$ (fourfold vertex). Let $i_{1}, i_{2}, i_{4}, i_{5}$ be the odd numbers, then from $(* *)$ we have

$$
\left[\begin{array}{lll}
i_{1} & i_{2} & i_{3} \\
i_{4} & i_{5} & i_{6}
\end{array}\right]_{q}=(-1)^{\frac{i_{1}+i_{2}+i_{4}+i_{5}}{2}+1}\left[\begin{array}{ccc}
i_{1} & i_{2} & i_{3} \\
i_{4} & i_{5} & i_{6}
\end{array}\right]_{-q} .
$$

Hence if $v$ is a switch-vertex, then the sign in $(* *)$ is plus, otherwise minus.

3) There are three odd and three even numbers in the color of $v$ (threefold vertex). Let $i_{1}, i_{2}, i_{3}$ be the even numbers, then from $(* *)$ we have

$$
\left[\begin{array}{lll}
i_{1} & i_{2} & i_{3} \\
i_{4} & i_{5} & i_{6}
\end{array}\right]_{q}=(-1)^{\frac{i_{1}+i_{2}+i_{3}}{2}+1}\left[\begin{array}{ccc}
i_{1} & i_{2} & i_{3} \\
i_{4} & i_{5} & i_{6}
\end{array}\right]_{-q} .
$$

By a cost of an edge $E$ we mean the half-sum of the numbers from the color of $E$. Let $E^{\prime}$ be a half-edge of an edge $E$. By a cost of the half-edge $E^{\prime}$ we mean the cost of $E$. 
We call an edge or a half-edge bad if its color is even (that is all three numbers in the color of the edge are even) and its cost is even. Let us show that the number of threefold vertices with a bad half-edge is even. It is sufficient to prove that the number of the bad half-edges incident to an even vertex is even, but this statement follows from the fact that the sum of all 4 costs of the half-edges incident to an even vertex is even. Hence we can think that for any threefold vertex the sign in $(* *)$ is plus.

Let us denote the number of the odd-colored edges of $X$ by $e$, the number of the threefold vertices by $n_{3}$, and the number of the fourfold vertices by $n_{4}$. Denote the number of the odd colored 2-components of $X$ by $c$. Then we have $c=\chi(S(\varphi))-n_{3}-n_{4}+e$. Hence

$$
|X, \varphi|_{q}=(-1)^{c+n_{4}-x+e}|X, \varphi|_{-q}=(-1)^{\chi(S(\varphi))-n_{3}-x}|X, \varphi|_{-q} .
$$

It is easy to see that for any admissible coloring $\varphi$ of a special polyhedron $X$ the number $n_{3}$ is even. This finishes the proof.

Let $S X$ be the set of singular points of $X$. Note that $S X$ is a regular graph of degree 4. Denote by $V$ the set of vertices of $X$, by $N(V, S X)$ a closed regular neighborhood of $V$ in $S X$, and by $N(V, X)$ a closed regular neighborhood of $V$ in $X$. The intersection of the union of the open edges with each connected component of $N(V, S X)$ consists of 4 half-open 1-cells, which are called thorns. The intersection of the union of the open 2-cells with each connected component of $N(V, X)$ consists of six half-open 2-cells, which are called wings.

Let $v$ be a vertex of $X$, and let $N(v, M)$ be a closed regular neighborhood of $v$. Choose a thorn $t$ in $N(v, M)$ and a small normal disk $D$ for it. Any orientation $\alpha$ of $N(v, M)$ induces an orientation $\left.\alpha\right|_{D}$ of $D$ according to the following convention: $\left.\alpha\right|_{D}$ together with the outward orientation of $t$ should give the orientation $\alpha$. Note that $\left.\alpha\right|_{D}$ induces a cyclic order on the set of wings adjacent to $t$.

Regular neighborhood $N(V, M)$ consists of 3-balls $N(v, M), \quad v \in V$. Choose orientations for the 3-balls. Let $E$ be an edge of $X$. It contains two thorns $t_{1}, t_{2}$. Let $W_{1}^{(i)}, W_{2}^{(i)}, W_{3}^{(i)}$ be the wings adjacent to $t_{i}$, where $i=1,2$. As above, the orientation of $N(V, M)$ induces a cyclic order on the set

$$
\left\{W_{1}^{(i)}, W_{2}^{(i)}, W_{3}^{(i)}\right\}, \quad \text { for } \quad i=1,2 .
$$

The 2-cells of $X$ determine the natural bijection

$$
f:\left\{W_{1}^{(1)}, W_{2}^{(1)}, W_{3}^{(1)}\right\} \rightarrow\left\{W_{1}^{(2)}, W_{2}^{(2)}, W_{3}^{(2)}\right\} .
$$

We shall say that the edge $E$ is odd if the bijection $f$ preserves the cyclic order on the wings, and even otherwise.

Theorem 2. Let $X$ be a special spine of a 3-manifold $M$. Then for any $\varphi \in \operatorname{Adm}(X)$ we have

$$
|X, \varphi|_{q}=(-1)^{\chi(S(\varphi))}|X, \varphi|_{-q}
$$

Proof. Let $x$ be the number of switch-vertices of the pair $(X, \varphi)$. By lemma 3 it is sufficient to prove that this number is even. 
Consider the coloring $\bar{\varphi}:\left\{\Gamma_{1}, \ldots, \Gamma_{b}\right\} \rightarrow \mathbf{Z}_{4}$ such that

for any $1 \leq i \leq b$.

$$
\bar{\varphi}\left(\Gamma_{i}\right)=\left\{\begin{array}{lll}
0, & \text { if } \varphi\left(\Gamma_{i}\right) \equiv 0 & (\bmod 2), \\
1, & \text { if } \varphi\left(\Gamma_{i}\right) \equiv 1 \quad(\bmod 4), \\
3, & \text { if } \varphi\left(\Gamma_{i}\right) \equiv 3 \quad(\bmod 4),
\end{array}\right.
$$

Fix an orientation of $N(V, M)$. Then each edge of $S X$ becomes odd or even. Let $G$ be the union of the edges of $X$ with the colors $\{0,1,3\}$ under the coloring $\bar{\varphi}$. Let $\Omega_{1}, \ldots, \Omega_{p}$ be the middle points of the odd edges of $G$. Consider a graph $G^{\prime}$. The set of vertices of $G^{\prime}$ consists of the vertices of $G$ and of the points $\Omega_{1}, \ldots, \Omega_{p}$. The set of edges of $G^{\prime}$ consists of the even edges of $G$ and of the halves of the odd edges of $G$. So each odd edge of $G$ gives 2 edges in $G^{\prime}$. The orientation of $N(V, M)$ and the coloring $\bar{\varphi}$ give the orientation of the graph $G^{\prime}$. Let $v_{1}, \ldots, v_{t}$ be the vertices of $G^{\prime}$. We will denote by $a_{i}$ the number of incoming and by $b_{i}$ the number of outgoing edges for the vertex $v_{i}$. We have $\left(a_{i}-b_{i}\right) \equiv 2 \quad(\bmod 4)$ iff $v_{i}$ is either the switch-vertex or the middle point of an odd edge, and $\left(a_{i}-b_{i}\right) \equiv 0 \quad(\bmod 4)$ otherwise. The number of vertices with the condition $\left(a_{i}-b_{i}\right) \equiv 2 \quad(\bmod 4)$ is even for any oriented graph, because $\sum_{i=1}^{t}\left(a_{i}-b_{i}\right)=0$.

It remains to prove that the number $p$ is even. Let $\theta$ be the number of odd edges of $X$ with the color $\{0,1,1\}$ under the coloring $\bar{\varphi}$. Then 1 -colored (by $\bar{\varphi}) 2$-cells pass $(2 \theta+p)$ times along the odd edges of $X$. Note that each 2-component of $X$ passes along the odd edges of $X$ even number of times (this is true for every special spine; see, for instance, $[\mathrm{F}])$. Therefore the number $(2 \theta+p)$ is even and $p$ is even.

Rem ark 4. In the case of an orientable 3-manifold this theorem was proved in [S1].

Corollary 3. For any 3-manifold $M$ and any $q$ we have

$$
\begin{aligned}
& T V_{N}(M)_{q}=(-1)^{N} T V_{N}(M)_{-q}, \quad \text { where } N \in\{0,1,2\}, \\
& T V_{0}(M)_{q}+T V_{2}(M)_{q}=\frac{1}{2}\left(T V(M)_{q}+T V(M)_{-q}\right), \\
& T V_{1}(M)_{q}=\frac{1}{2}\left(T V(M)_{q}-T V(M)_{-q}\right) .
\end{aligned}
$$

Remark 5. In the papers [S1] and [S2] we used the parameter $-\bar{q}$ instead of $-q$, but it is easy to see that $[n]_{q}=[n]_{\bar{q}}$.

7. The tables. Below we present the summand-invariants $T V_{N}(M)_{q}$ and the TuraevViro invariants $T V^{*}(M)_{q}$ with $r \leq 7$ for the manifolds $S^{3}, \mathbf{R} P^{3}, L_{3,1}, L_{4,1}, L_{5,1}, L_{5,2}, L_{6,1}$, $L_{7,2}, L_{8,3}, L_{9,2}, L_{10,3}, L_{11,4}, L_{12,5}, L_{13,5}, S^{3} / Q_{8}, S^{3} / Q_{12}$, where $S^{3} / G$ denotes the quotient space of the sphere $S^{3}$ by a linear free action of a finite nonabelian group $G$. These are all closed irreducible orientable 3 -manifolds, having a special spine with $\leq 3$ vertices.

Each summand invariant is presented by a polynomial on $q$ (here $q$ is a primitive root of unity of degree $2 r$ ) with integer coefficients, and by evaluation of the polynomial at $q=e^{i \pi / r}$. Note that each coefficient in the polynomial is a separate invariant. The invariants from the tables are related by the equality

$$
T V^{*}(M)_{q}=-\frac{\left(q-q^{-1}\right)^{2}}{2 r}\left(T V_{0}(M)_{q}+T V_{1}(M)_{q}+T V_{2}(M)_{q}\right) .
$$


Table 1: Invariants for $S^{3}$

\begin{tabular}{llllllll}
$r$ & $T V_{0}(M)_{q}$ & & $T V_{1}(M)_{q}$ & & $T V_{2}(M)_{q}$ & & $T V^{*}(M)_{q}$ \\
\hline 3 & 1 & $=1.000$ & 0 & $=0.000$ & 0 & $=0.000$ & 0.500 \\
4 & 1 & $=1.000$ & 0 & $=0.000$ & 0 & $=0.000$ & 0.250 \\
5 & 1 & $=1.000$ & 0 & $=0.000$ & 0 & $=0.000$ & 0.138 \\
6 & 1 & $=1.000$ & 0 & $=0.000$ & 0 & $=0.000$ & 0.083 \\
7 & 1 & $=1.000$ & 0 & $=0.000$ & 0 & $=0.000$ & 0.054
\end{tabular}

Table 2: Invariants for $\mathbf{R} P^{3}$

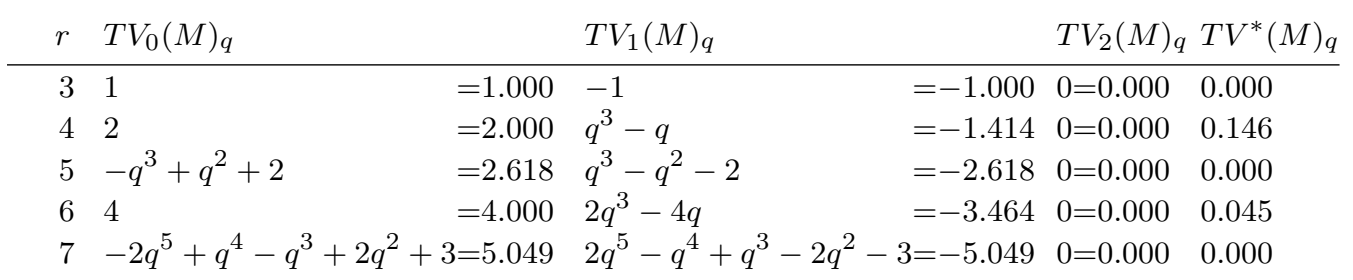

Table 3: Invariants for $L_{3,1}$

\begin{tabular}{llllllll}
$r$ & $T V_{0}(M)_{q}$ & & $T V_{1}(M)_{q}$ & & $T V_{2}(M)_{q}$ & & $T V^{*}(M)_{q}$ \\
\hline 3 & 1 & $=1.000$ & 0 & $=0.000$ & 0 & $=0.000$ & 0.500 \\
4 & 1 & $=1.000$ & 0 & $=0.000$ & 0 & $=0.000$ & 0.250 \\
5 & $-q^{3}+q^{2}+2$ & $=2.618$ & 0 & $=0.000$ & 0 & $=0.000$ & 0.362 \\
6 & 3 & $=3.000$ & 0 & $=0.000$ & 0 & $=0.000$ & 0.250 \\
7 & $-q^{5}+q^{2}+2$ & $=3.247$ & 0 & $=0.000$ & 0 & $=0.000$ & 0.175
\end{tabular}

Table 4: Invariants for $L_{4,1}$

\begin{tabular}{llllllll}
$r$ & $T V_{0}(M)_{q}$ & & $T V_{1}(M)_{q}$ & $T V_{2}(M)_{q}$ & & $T V^{*}(M)_{q}$ \\
\hline 3 & 1 & $=1.000$ & 0 & $=0.000$ & 1 & $=1.000$ & 1.000 \\
4 & 2 & $=2.000$ & 0 & $=0.000$ & 0 & $=0.000$ & 0.500 \\
5 & 1 & $=1.000$ & 0 & $=0.000$ & 1 & $=1.000$ & 0.276 \\
6 & 4 & $=4.000$ & 0 & $=0.000$ & 0 & $=0.000$ & 0.333 \\
7 & $-q^{5}+q^{2}+2$ & $=3.247$ & 0 & $=0.000$ & $-q^{5}+q^{2}+2$ & $=3.247$ & 0.349
\end{tabular}

Table 5: Invariants for $L_{5,1}$

\begin{tabular}{|c|c|c|c|c|c|c|c|}
\hline$r$ & $T V_{0}(M)_{q}$ & & & I) $q$ & & $M)_{q}$ & $T V^{*}(M)_{q}$ \\
\hline 3 & 1 & $=1.000$ & 0 & $=0.000$ & 0 & $=0.000$ & 0.500 \\
\hline 4 & 1 & $=1.000$ & 0 & $=0.000$ & 0 & $=0.000$ & 0.250 \\
\hline 5 & $-q^{3}+q^{2}+3$ & $=3.618$ & 0 & $=0.000$ & 0 & $=0.000$ & 0.500 \\
\hline 6 & 1 & $=1.000$ & 0 & $=0.000$ & 0 & $=0.000$ & 0.083 \\
\hline 7 & $-2 q^{5}+q^{4}-q^{3}+2 q^{2}+$ & $3=5.049$ & 0 & $=0.000$ & 0 & $=0.000$ & 0.272 \\
\hline
\end{tabular}


Table 6: Invariants for $L_{5,2}$

\begin{tabular}{llllllll}
$r$ & $T V_{0}(M)_{q}$ & & $T V_{1}(M)_{q}$ & \multicolumn{2}{l}{$T V_{2}(M)_{q}$} & $T V^{*}(M)_{q}$ \\
\hline 3 & 1 & $=1.000$ & 0 & $=0.000$ & 0 & $=0.000$ & 0.500 \\
4 & 1 & $=1.000$ & 0 & $=0.000$ & 0 & $=0.000$ & 0.250 \\
5 & 0 & $=0.000$ & 0 & $=0.000$ & 0 & $=0.000$ & 0.000 \\
6 & 1 & $=1.000$ & 0 & $=0.000$ & 0 & $=0.000$ & 0.083 \\
7 & $-2 q^{5}+q^{4}-q^{3}+2 q^{2}+3$ & $=5.049$ & 0 & $=0.000$ & 0 & $=0.000$ & 0.272
\end{tabular}

Table 7: Invariants for $L_{6,1}$

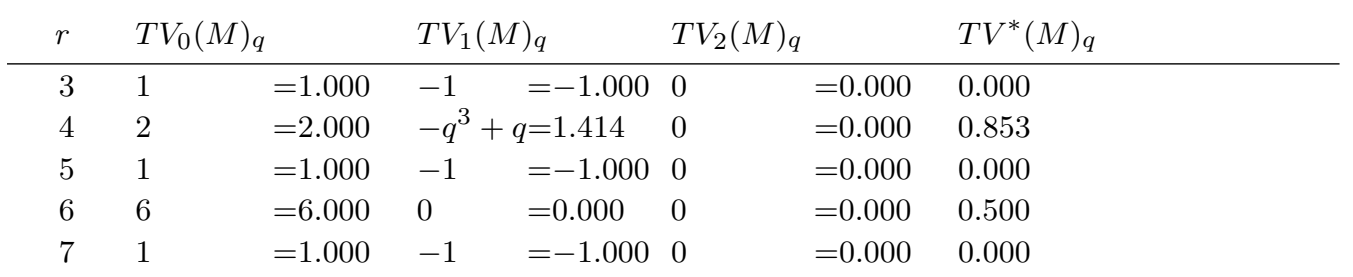

Table 8: Invariants for $L_{7,2}$

\begin{tabular}{|c|c|c|c|c|c|c|}
\hline$r$ & $T V_{0}(M)_{q}$ & \multicolumn{2}{|c|}{$T V_{1}(M)_{q}$} & \multicolumn{2}{|c|}{$T V_{2}(M)_{q}$} & $T V^{*}(M)_{q}$ \\
\hline 3 & $=1.000$ & 0 & $=0.000$ & 0 & $=0.000$ & 0.500 \\
\hline 4 & $=1.000$ & 0 & $=0.000$ & 0 & $=0.000$ & 0.250 \\
\hline 5 & $-q^{3}+q^{2}+2=2.618$ & 0 & $=0.000$ & 0 & $=0.000$ & 0.362 \\
\hline 6 & $=1.000$ & 0 & $=0.000$ & 0 & $=0.000$ & 0.083 \\
\hline 7 & $=0.000$ & 0 & $=0.000$ & 0 & $=0.000$ & 0.000 \\
\hline
\end{tabular}

Table 9: Invariants for $L_{8,3}$

\begin{tabular}{|c|c|c|c|c|c|c|}
\hline$r$ & $T V_{0}(M)_{q}$ & \multicolumn{2}{|c|}{$T V_{1}(M)_{q}$} & \multicolumn{2}{|c|}{$T V_{2}(M)_{q}$} & $T V^{*}(M)_{q}$ \\
\hline 3 & $=1.000$ & 0 & $=0.000$ & 1 & $=1.000$ & 1.000 \\
\hline 4 & $=2.000$ & 0 & $=0.000$ & 2 & $=2.000$ & 1.000 \\
\hline 5 & $-q^{3}+q^{2}+2=2.618$ & 0 & $=0.000$ & & $2=2.618$ & 0.724 \\
\hline 6 & $=4.000$ & 0 & $=0.000$ & 0 & $=0.000$ & 0.333 \\
\hline 7 & $=1.000$ & 0 & $=0.000$ & 1 & $=1.000$ & 0.108 \\
\hline
\end{tabular}

Table 10: Invariants for $L_{9,2}$

\begin{tabular}{|c|c|c|c|c|c|c|c|}
\hline$r$ & $T V_{0}(M)_{q}$ & & & & & & $T V^{*}(M)_{q}$ \\
\hline 3 & 1 & $=1.000$ & 0 & $=0.000$ & 0 & $=0.000$ & 0.500 \\
\hline 4 & 1 & $=1.000$ & 0 & $=0.000$ & 0 & $=0.000$ & 0.250 \\
\hline 5 & 1 & $=1.000$ & 0 & $=0.000$ & 0 & $=0.000$ & 0.138 \\
\hline 6 & 3 & $=3.000$ & 0 & $=0.000$ & 0 & $=0.000$ & 0.250 \\
\hline 7 & $-2 q^{5}+q^{4}-q^{3}+2 q^{2}+$ & $3=5.049$ & 0 & $=0.000$ & 0 & $=0.000$ & 0.272 \\
\hline
\end{tabular}


Table 11: Invariants for $L_{10,3}$

\begin{tabular}{llllllll}
$r$ & $T V_{0}(M)_{q}$ & & $T V_{1}(M)_{q}$ & & $T V_{2}(M)_{q}$ & $T V^{*}(M)_{q}$ \\
\hline 3 & 1 & $=1.000$ & -1 & $=-1.000$ & 0 & $=0.000$ & 0.000 \\
4 & 2 & $=2.000$ & $-q^{3}+q$ & $=1.414$ & 0 & $=0.000$ & 0.853 \\
5 & 0 & $=0.000$ & 0 & $=0.000$ & 0 & $=0.000$ & 0.000 \\
6 & 4 & $=4.000$ & $-2 q^{3}+4 q$ & $=3.464$ & 0 & $=0.000$ & 0.622 \\
7 & $-q^{5}+q^{2}+2$ & $=3.247$ & $q^{5}-q^{2}-2$ & $=-3.247$ & 0 & $=0.000$ & 0.000
\end{tabular}

Table 12: Invariants for $L_{11,4}$

\begin{tabular}{llllllll}
$r$ & $T V_{0}(M)_{q}$ & & $T V_{1}(M)_{q}$ & & $T V_{2}(M)_{q}$ & $T V^{*}(M)_{q}$ \\
\hline 3 & 1 & $=1.000$ & 0 & $=0.000$ & 0 & $=0.000$ & 0.500 \\
4 & 1 & $=1.000$ & 0 & $=0.000$ & 0 & $=0.000$ & 0.250 \\
5 & 1 & $=1.000$ & 0 & $=0.000$ & 0 & $=0.000$ & 0.138 \\
6 & 1 & $=1.000$ & 0 & $=0.000$ & 0 & $=0.000$ & 0.083 \\
7 & $-q^{5}+q^{2}+2=3.247$ & 0 & $=0.000$ & 0 & $=0.000$ & 0.175
\end{tabular}

Table 13: Invariants for $L_{12,5}$

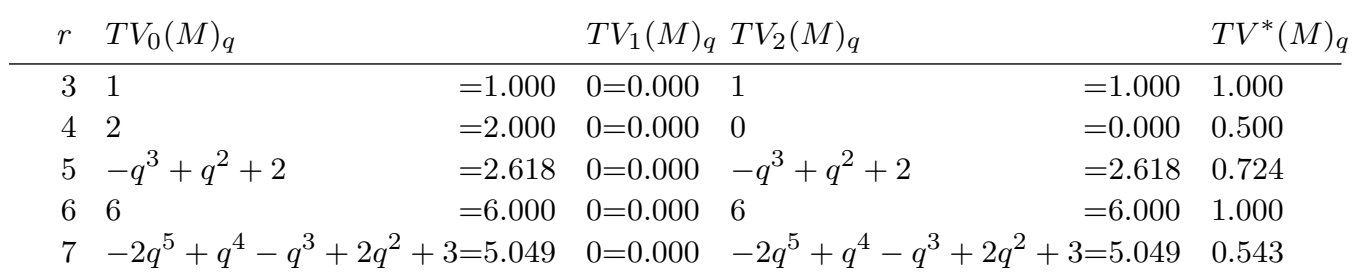

Table 14: Invariants for $L_{13,5}$

\begin{tabular}{llllllll}
$r$ & $T V_{0}(M)_{q}$ & & $T V_{1}(M)_{q}$ & & $T V_{2}(M)_{q}$ & & $T V^{*}(M)_{q}$ \\
\hline 3 & 1 & $=1.000$ & 0 & $=0.000$ & 0 & $=0.000$ & 0.500 \\
4 & 1 & $=1.000$ & 0 & $=0.000$ & 0 & $=0.000$ & 0.250 \\
5 & $-q^{3}+q^{2}+2=2.618$ & 0 & $=0.000$ & 0 & $=0.000$ & 0.362 \\
6 & 1 & $=1.000$ & 0 & $=0.000$ & 0 & $=0.000$ & 0.083 \\
7 & 1 & $=1.000$ & 0 & $=0.000$ & 0 & $=0.000$ & 0.054
\end{tabular}

Table 15: Invariants for $S^{3} / Q_{8}$

\begin{tabular}{|c|c|c|c|c|c|c|}
\hline$r$ & $T V_{0}(M)_{q}$ & & & & $T V_{2}(M)_{q}$ & $T V^{*}(M)_{q}$ \\
\hline 3 & 1 & $=1.000$ & 0 & $=0.000$ & $=3.000$ & 2.000 \\
\hline 4 & 4 & $=4.000$ & 0 & $=0.000$ & $=6.000$ & 2.500 \\
\hline 5 & $-q^{3}+q^{2}+4$ & $=4.618$ & 0 & $=0.000$ & $-3 q^{3}+3 q^{2}+12=13.854$ & 2.553 \\
\hline 6 & 10 & $=10.000$ & 0 & $=0.000$ & $=18.000$ & 2.333 \\
\hline 7 & $-2 q^{5}+2 q^{2}+7$ & $7=9.494$ & 0 & $=0.000$ & $-6 q^{5}+6 q^{2}+21=28.482$ & 2.043 \\
\hline
\end{tabular}


Table 16: Invariants for $S^{3} / Q_{12}$

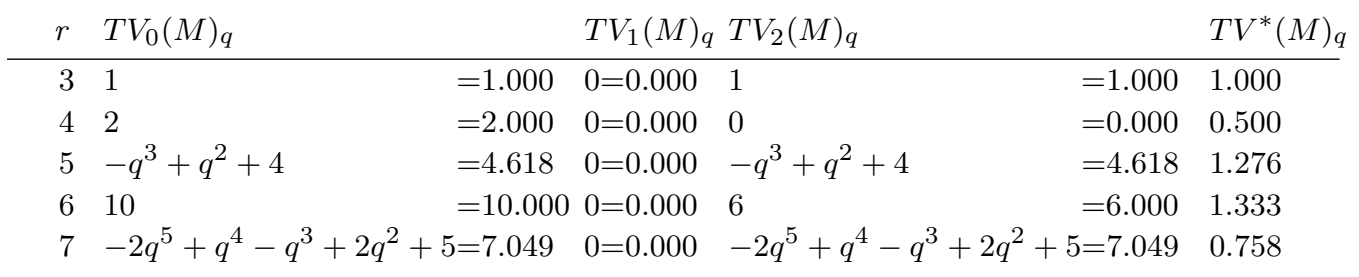

\section{References}

[BHMV] C. Blanchet, N. Habegger, G. Masbaum, P. Vogel, Remarks on the threemanifold invariants $\theta_{p}$, 'Operator Algebras, Mathematical Physics, and Low Dimensional Topology' (Nato Workshop July 1991), Vol. 5, 39-59.

[Ca] B. G. Casler, An imbedding theorem for connected 3-manifolds with boundary, Math. Soc. 16 (1965), 559-566.

[F] A. T. Fomenko, Visual geometry and topology, Springer-Verlag, Berlin and Heidelberg, 1994.

[KL1] L. H. Kauffman and S. Lins, Computing Turaev-Viro invariants for 3-manifolds, Manuscripta Math. 72 (1991), 81-94.

[KL2] L. H. Kauffman and S. Lins, Temperley-Lieb recoupling theory and invariants of 3-manifolds, Princeton University Press, Princeton, N. J., 1994.

$[\mathrm{KM}] \quad$ R. C. Kirby, P. Melvin, The 3-manifold invariants of Witten and ReshetikhinTuraev for $\operatorname{sl}(2, \mathbf{C})$, Inv. Math. 105 (1991), 473-545.

[M] S. V. Matveev, Transformations of special spines and the Zeeman conjecture, Math. USSR Izvestia, Vol. 31, 2, 1988, 423-434.

[N] J. R. Neil, Combinatorial calculation of the various normalizations of the Witten invariants for 3-manifolds, J. of Knot Theory and Its Ramifications, Vol. 1, 4 (1992), 407-449.

[P] R. Piergallini, Standard moves for standard polyhedra and spines, Rend. Circ. Mat. Palermo, Vol. 37, 18 (1988), 391-414.

[S1] M. Sokolov, On Turaev-Viro invariants for 3-manifolds, VINITI preprint 583-B93 (in Russian).

[S2] M. Sokolov, The Turaev-Viro invariant for 3-manifolds is a sum of three invariants, Canad. Math. Bull., Vol. 39(4), 1996, pp 468-475.

[T1] V. G. Turaev, Topology of shadows. Preprint, 1991.

[T2] V. G. Turaev, Quantum invariants of knots and 3-manifolds, Walter de Gruyter, Berlin, New York, 1994.

[TV] V. G. Turaev and O. Y. Viro, State sum invariants of 3-manifolds and quantum 6j-symbols, Topology, 31 (1992), 866-902.

[W] K. Walker, On Witten's 3-manifold invariants, Preprint, 1991. 